\title{
The molecular basis of
}

\section{frontotemporal dementia}

\section{Manuela Neumann ${ }^{1}$, Markus Tolnay ${ }^{2}$ and lan R.A. Mackenzie ${ }^{3, *}$}

Frontotemporal dementia (FTD) is a clinical syndrome with a heterogeneous molecular basis. Familial FTD has been linked to mutations in several genes, including those encoding the microtubule-associated protein tau (MAPT), progranulin (GRN), valosin-containing protein (VCP) and charged multivescicular body protein 2B (CHMP2B). The associated neuropathology is characterised by selective degeneration of the frontal and temporal lobes (frontotemporal lobar degeneration, FTLD), usually with the presence of abnormal intracellular protein accumulations. The current classification of FTLD neuropathology is based on the identity of the predominant protein abnormality, in the belief that this most closely reflects the underlying pathogenic process. Major subgroups include those characterised by the pathological tau, TDP-43, intermediate filaments and a group with cellular inclusions composed of an unidentified ubiquitinated protein. This review will focus on the current understanding of the molecular basis of each of the major FTLD subtypes. It is anticipated that this knowledge will provide the basis of future advances in the diagnosis and treatment of FTD.

The clinical syndrome of frontotemporal dementia (FTD) is characterised by progressive changes in behaviour, personality and/or language, with relative preservation of memory (Refs 1, 2, 3). Clinical subtypes include the frontal (behavioural) variant (fvFTD) and two forms of primary progressive aphasia (PPA): primary non-fluent aphasia (PNFA) and semantic dementia (SD) (Refs 1, 2). FTD is often associated with an extrapyramidal movement disorder (parkinsonism or corticobasal syndrome) or with motor neuron disease (MND) (Refs 4, 5). FTD accounts for $5-15 \%$ of all dementia and is the second commonest cause in the presenile age group (Refs 6, 7). A family history of similar disease is present in $25-50 \%$ of patients, indicating a significant genetic influence (Refs 8, 9, 10).

It should be noted that there are currently two conventions used for the nomenclature of this clinicopathological syndrome. Here, we use the one that is more popular in North America, in

\footnotetext{
${ }^{1}$ Institute of Neuropathology, University Hospital of Zürich, Switzerland.

${ }^{2}$ Institute of Pathology, Department of Neuropathology, University of Basel, Switzerland.

${ }^{3}$ Department of Pathology, University of British Columbia, Vancouver, Canada.

*Corresponding author: Ian R.A. Mackenzie, Department of Pathology, Vancouver General Hospital, 855 West 12th Avenue, Vancouver, British Columbia, V5Z 1M9, Canada. Tel: +1 604875 4480; Fax: +1 604875 5707; E-mail: ian.mackenzie@vch.ca
} 
which FTD is used as the general term for the clinical syndrome (including all behavioural and language variants) and the term frontotemporal lobar degeneration (FTLD) is reserved for the associated pathology. However, in the UK, FTLD is often used as the general term for both the clinical and the pathological entities, whereas FTD refers only to the clinical subtype in which behavioural abnormalities predominate. We recognise that this situation is confusing; however, both terminologies are currently in wide use and there is no consensus as to which is more appropriate.

The molecular genetic basis of FTD is heterogeneous. Autosomal dominant FTD may be caused by mutations in several genes, including those encoding the microtubuleassociated protein tau (MAPT) (Refs 11, 12, 13), progranulin (GRN) (Refs 14, 15), valosincontaining protein $(V C P)$ (Ref. 16) and charged multivescicular body protein 2B (CHMP2B) (Ref. 17), and several families with FTD and MND have shown genetic linkage to a locus on chromosome 9p (Refs 18, 19, 20).

The neuropathology associated with clinical FTD is also heterogeneous (Ref. 21). Relatively selective degeneration of the frontal and temporal lobes (frontotemporal lobar degeneration, FTLD) is a consistent feature, which correlates with the main clinical manifestations. As with many other neurodegenerative conditions, the pathology of most cases of FTD includes the presence of abnormal intracellular protein accumulations. Traditionally, these inclusion bodies were demonstrated with special histochemical staining techniques, such as silver impregnation methods. Eponymous and syndromic names were used for clinical syndromes associated with a specific morphology and anatomical distribution of cellular inclusions. However, in many cases, these clinicopathological correlations turned out to be imperfect. Modern laboratory techniques, such as immunohistochemistry, have allowed the biochemical composition of the pathological changes to be more readily determined. In recent years, it has become popular to classify FTLD pathology based on the presumed molecular defect, in the belief that this most closely reflects the underlying pathogenic process (Ref. 21).

In this review, we will use the molecular-based system of nosology and nomenclature recommended in a recent consensus paper (Ref. 22). The term FTLD will be used as the general terminology for pathological conditions that are commonly associated with clinical FTD, and major subdivisions will be designated by the protein abnormality that is presumed to be pathogenic or most characteristic (Table 1). Cases are further subclassified, using traditional terminology, to define specific patterns of pathology [i.e. FTLD-tau (PiD) for Pick disease]. Existing descriptive terms are retained for rare causes of FTD that have characteristic pathological features of unknown biochemistry (such as basophilic inclusion body disease, BIBD). Finally, cases with no inclusions visible with special histochemical stains or immunohistochemistry (formerly known as dementia lacking distinctive histopathology, DLDH), will be designated FTLD-ni (no inclusions).

\section{Tau protein}

\section{FTLD-tau}

Tau is a microtubule-associated phosphoprotein (MAP) that is abundantly expressed in both the central and peripheral nervous system. By promoting microtubule (MT) assembly and stability, tau plays a fundamental role in maintaining neuronal integrity and axoplasmic transport (Refs 23, 24). The tau gene (MAPT) is located on chromosome $17 \mathrm{q} 21$ and has two major haplotypes, $\mathrm{H} 1$ and $\mathrm{H} 2$, which are defined by a set of single nucleotide polymorphisms and a 238 base pair deletion in intron 9 (Ref. 25). The $\mathrm{H} 2$ haplotype is also associated with an inversion of a $\sim 900 \mathrm{~kb}$ region that includes MAPT (Ref. 26). MAPT contains 16 exons, 11 of which encode the major tau protein isoforms (Ref. 27). Through alternative mRNA splicing of exons 2, 3 and 10, a set of six isoforms, ranging from 352 to 441 amino acids, are generated in the adult human brain. Exons 9 to 12 encode four microtubulebinding motifs, which are imperfect repeats of 31 or 32 amino acids, in the C-terminal half of the tau molecule. It is these binding domains that mediate the interaction between tau and MTs. Alternative splicing of exon 10 generates tau isoforms with either three (exon 10 missing) or four (exon 10 present) repeat domains, known as $3 \mathrm{R}$ and $4 \mathrm{R}$ tau, respectively (Fig. 1a) (Refs 28, 29). In Alzheimer disease (AD), abnormally hyperphosphorylated tau is the major component of the neurofibrillary lesions (known as neurofibrillary tangles, neuropil 
Table 1. Recommended nomenclature for frontotemporal lobar degenerations

\begin{tabular}{|c|c|c|c|c|}
\hline \multicolumn{2}{|c|}{ Previous terminology } & $\begin{array}{l}\text { Recommended } \\
\text { terminology } \\
\text { (Ref. 13) }\end{array}$ & $\begin{array}{l}\text { Major } \\
\text { pathological } \\
\text { subtypes }^{\mathrm{a}}\end{array}$ & $\begin{array}{l}\text { Associated } \\
\text { gene }\end{array}$ \\
\hline \multicolumn{2}{|l|}{ Tau-positive FTLD } & FTLD-tau & $\begin{array}{l}\text { PiD } \\
\text { CBD } \\
\text { PSP } \\
\text { AgD } \\
\text { MSTD } \\
\text { FTDP-17T }\end{array}$ & MAPT \\
\hline \multirow[t]{5}{*}{ Tau-negative FTLD } & $\begin{array}{l}\text { FTLD-U, TDP-43- } \\
\text { positive }\end{array}$ & FTLD-TDP & $\begin{array}{l}\text { Type } 1^{\mathrm{C}} \\
\text { Type } 2^{\mathrm{c}} \\
\text { Type } 3^{\mathrm{C}} \\
\text { Type } 4^{\mathrm{C}}\end{array}$ & $\begin{array}{l}\text { Chrom. 9p } \\
\text { GRN } \\
\text { VCP }\end{array}$ \\
\hline & $\begin{array}{l}\text { FTLD-U, TDP-43- } \\
\text { negative }\end{array}$ & FTLD-UPS & $\begin{array}{l}\text { aFTLD-U } \\
\text { FTD-3 }\end{array}$ & CHMP2B \\
\hline & NIFID & FTLD-IF & & \\
\hline & DLDH & FTLD-ni & & \\
\hline & Other (BIBD) & BIBD & & \\
\hline \multicolumn{5}{|c|}{$\begin{array}{l}{ }^{a} \text { Characteristic pattern of pathology, not the clinical syndrome. } \\
\text { bFTDP-17T is not really a pathological subtype because the pathology varies between cases with different MAPT } \\
\text { mutations. } \\
\text { 'Based on classification system by Cairns et al. (Ref. 100). } \\
\text { Abbreviations: aFTLD-U, atypical frontotemporal lobar degeneration with ubiquitinated inclusions; AgD, } \\
\text { argyrophilic grain disease; BIBD, basophilic inclusion body disease; CBD, corticobasal degeneration; CHMP2B, } \\
\text { charged multivescicular body protein 2B gene; chrom. 9p, linked to locus on chromosome 9p; DLDH, dementia } \\
\text { lacking distinctive histopathology; FTD-3, frontotemporal dementia linked to chromosome 3; FTLD, } \\
\text { frontotemporal lobar degeneration; FTLD-U, FTLD with ubiquitinated inclusions; FTDP-17T, frontotemporal } \\
\text { dementia and parkinsonism caused by mutations in the tau gene; GRN, progranulin gene; IF, intermediate } \\
\text { filament; } M A P T \text {, microtubule-associated protein tau gene; MSTD, multiple system tauopathy with dementia; ni, } \\
\text { no inclusions; NIFID, neuronal intermediate filament inclusion disease; NII, neuronal intranuclear inclusion; PiD, } \\
\text { Pick disease; PSP, progressive supranuclear palsy; UPS, ubiquitin proteasome system; VCP, gene encoding } \\
\text { valosin-containing protein. }\end{array}$} \\
\hline
\end{tabular}

threads and dystrophic neurites), whereas $\beta$ amyloid peptide is the major component of senile plaques. Abnormal intracellular accumulation of tau is also characteristic of a number of other neurodegenerative disorders, collectively known as 'tauopathies' (Refs 30, 31). Comparison of the tau aggregates in these disorders reveals differences in both phosphorylation and the content of different tau isoforms. Thus, tauopathies might be subdivided into disorders with inclusions made predominantly of $3 R$ or $4 R$ tau or an admixture of both (Ref. 32). A number of these tauopathies may be associated with clinical FTD (FTLDtau), including Pick disease (PiD), progressive supranuclear palsy (PSP), corticobasal degeneration (CBD), argyrophilic grain disease (AGD) and hereditary frontotemporal dementia and parkinsonism linked to chromosome 17, as a result of MAPT mutations (FTDP-17T) (Ref. 21).

\section{FTLD-tau (PiD)}

PiD is the clinicopathological prototype of FTD (Refs 33, 34, 35). The most distinctive gross pathological feature is marked atrophy of the frontal and anterior temporal lobes, also known as 'knife-edge' lobar atrophy (Fig. 2a). The main histological features consist of severe neuronal loss, ballooned neurons (Pick cells) and pathognomonic spherical argyrophilic neuronal inclusions, called Pick bodies (PBs) (Fig. 2b). PBs are found mainly in 
Figure 1. Tau isoforms and tau gene mutations. (a) Six isoforms of the tau protein found in the adult human brain result from alternative splicing of exons 2 (red), 3 (green) and 10 (yellow). Exons 9-12 encode imperfectly repeated microtubule-binding repeats (shown in black). (b) Mutations in the tau gene in frontotemporal dementia and parkinsonism linked to chromosome 17 (FTDP-17T). Thirty-six coding region mutations in exons (E) 1 and 9-13 and eight intronic mutations flanking exon 10 are shown.

neocortical neurons of layers II-IV, the granule cells of the dentate gyrus, pyramidal cells of the hippocampus, subiculum and entorhinal cortex, and subcortical nerve cell populations. PBs are composed predominantly of $3 R$ tau isoforms (Ref. 32), whereas $4 R$ tau deposits might be present in glia in a subset of cases (Refs 36, 37).

\section{FTLD-tau (PSP)}

Also known as Steele-Richardson-Olszewski syndrome (Ref. 38), PSP is the second mostcommon parkinsonian disorder, after classical Parkinson disease. Clinical features typically include early postural instability, axial dystonia, supranuclear vertical gaze palsy, pseudobulbar palsy and parkinsonism that is resistant to levodopa therapy. Although cognitive dysfunction is often mild, some cases of PSP present with dementia and only mild motor features (Ref. 39). The pathology of PSP is characterised by gross atrophy of the midbrain (especially the pretectal region), superior cerebellar peduncles, subthalamic nucleus and substantia nigra. Fibrillar tau pathology occurs in both neurons and glia. Glial inclusions include tufted astrocytes (Fig. 2c), which are fairly specific for PSP, as well as thorn-shaped astrocytes, coiled bodies in oligodendrocytes and neuropil threads, all of which are also 

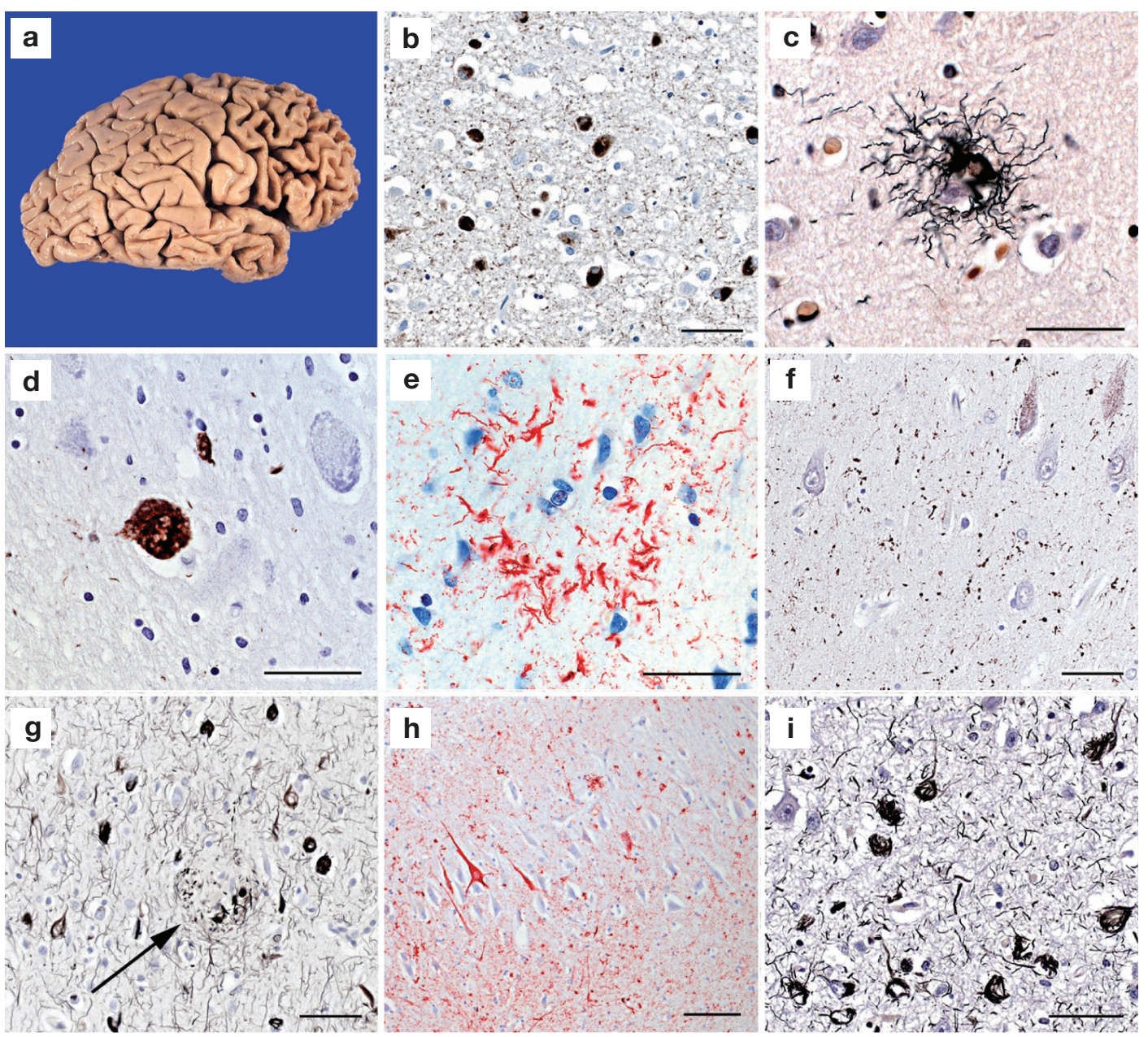

\section{Pathological features in FTLD-tau \\ Expert Reviews in Molecular Medicine @ 2009 Cambridge University Press}

Figure 2. Pathological features in FTLD-tau. (a) Severe atrophy of the frontal and temporal lobes and (b) Pick bodies in the temporal cortex of a patient with Pick disease. (c) Tufted astrocyte and (d) a globose tangle in a case with progressive supranuclear palsy. (e) Astrocytic plaque as a hallmark lesion of corticobasal degeneration. (f) Abundant argyrophilic grains in the hippocampus of a patient with argyrophilic grain disease. (g) Neurofibrillary tangles and neuropil threads in the frontal cortex of a case with frontal variant Alzheimer disease. Arrow indicates a neuritic plaque. (h) Tau pathology in sector CA2 of the hippocampus in hippocampal sclerosis dementia. (i) Neuronal and glial tau pathology in the frontal cortex of a patient with an intron $10+3$ splice-site mutation in the tau gene. b, d, e, f, h, tau immunohistochemistry; c, g, i, Gallyas-Braak silver stain. Scale bars: $50 \mu \mathrm{m}$.

found in other tauopathies. Neurofibrillary tangles typically have a round or globose appearance (Fig. 2d). Many subcortical regions are affected, including the striatum, pallidum, subthalamic nucleus, substantia nigra, oculomotor complex, periaqueductal grey, superior colliculi, basis pontis and dentate nucleus. Cases of PSP with dementia are also found to have significant tau pathology in the cerebral cortex (Ref. 40). Biochemically, the inclusions in PSP consist predominantly of $4 \mathrm{R}$ tau isoforms (Ref. 41). Inheritance of the H1 tau gene haplotype predisposes to the sporadic $4 \mathrm{R}$ tauopathies, PSP and CBD (Refs 42, 43).

\section{FTLD-tau (CBD)}

$\mathrm{CBD}$ is an increasingly recognised neurodegenerative disease with both motor and 
cognitive dysfunction (Ref. 44). The initial motor symptoms are akinesia, rigidity and apraxia. There is often parkinsonism, which is resistant to dopaminergic drugs. Recent studies have found that FTD is also a common clinical presentation of CBD (Refs 39, 45). Gross examination typically shows depigmentation of the substantia nigra and frontoparietal atrophy that is most severe in the pre- and post-central areas and often asymmetrical. Swollen, achromatic neurons were initially thought to be the most characteristic histological change (Ref. 44); however, it is now recognised that the affected cortex and subcortical regions have abundant glial and neuronal intracytoplasmic tau pathology. In addition to various neuronal inclusions, abundant thread pathology, coiled bodies and thorn-shaped astrocytes, a particularly characteristic feature of CBD is the presence of circular rings of short tau-positive cell processes, referred to as 'astrocytic plaques' (Fig. 2e) (Ref. 46). The biochemical profile of the pathological tau in $\mathrm{CBD}$ is similar to that in PSP, and consists predominantly of $4 \mathrm{R}$ tau isoforms (Refs 30, 31).

\section{FTLD-tau (AGD)}

AGD is a sporadic late-onset dementia that accounts for approximately 5\% of all cases of dementia (Refs 47,48). The clinical features are still poorly understood, and it can be difficult to distinguish from AD (Refs 49, 50). However, a subset of AGD patients present with clinical features of FTD (Refs 51, 52). The hallmark lesions are abundant argyrophilic grains (AGs) in neuronal processes (Fig. 2f) and coiled bodies in oligodendrocytes. AGs are abundant in various limbic structures, including the CA1 sector of the hippocampus, layers II and III of the entorhinal and transentorhinal cortices, the amygdala and the hypothalamic lateral tuberal nuclei (Ref. 47). Recent biochemical studies have revealed that AGD is also a $4 \mathrm{R}$ tauopathy (Refs 53, 54, 55). At present, there is controversy over whether the $\mathrm{H} 1$ tau haplotype is also a risk factor for AGD (Ref. 47).

\section{Other sporadic tauopathies}

Some additional rare forms or variants of tauopathies may be associated with clinical FTD and/or demonstrate a frontotemporal lobar distribution of tau pathology. These include the frontal variant of AD (fvAD) (Fig. 2g) (Refs 56,
57), neurofibrillary tangle dementia (reviewed in Ref. 58), sporadic multiple system tauopathy with dementia (MSTD) (Ref. 59), and white matter tauopathy with globular inclusions (Ref. 60). Although insoluble tau in fvAD and neurofibrillary tangle dementia contains both $3 R$ and $4 R$ tau isoforms, sporadic MSTD and white matter tauopathy with globular glial inclusions are $4 \mathrm{R}$ tauopathies. Hippocampal sclerosis (HS) is a descriptive term for nearcomplete, selective loss of pyramidal neurons in the CA1 sector of the hippocampus and subiculum. HS can be the primary pathology in some cases of late-onset dementia (Ref. 61), but it more frequently occurs in association with tauopathies (Fig. 2h) (Ref. 62) or FTLDTDP (see below) (reviewed in Ref. 63).

\section{FTDP-17T}

In 1994, a familial form of FTD and parkinsonism was linked to chromosome 17q21-22 (Ref. 64). Over the following years, other families with autosomal dominant FTD were identified with linkage to the same region and, based on similarities in the clinical, neuropathological, and genetic findings, these were grouped under the umbrella of FTD and parkinsonism linked to chromosome 17 (FTDP-17) (Ref. 65). About half of the original FTDP-17 families were found to have MAPT mutations (FTDP-17T) (Refs 11, 12, 13), whereas the genetic cause in the others remained unknown until recently (see section on FTLD-TDP). At present, 44 MAPT mutations have been described in 131 families (Fig. 1b) (AD\&FTD Mutation database: http://www.molgen.ua.ac.be/FTDMutations). Clinically, FTDP-17T is typically characterised by personality changes, motor symptoms and cognitive decline. The earliest symptoms are usually disinhibition, loss of initiative, obsessive-compulsive behaviour, and/or psychosis, followed by cognitive decline leading to profound dementia (Ref. 66). However, some MAPT mutations result in a clinical phenotype that more closely resembles PiD, CBD, PSP, AGD or AD. In all cases of FTDP-17T, the neuropathology includes various types of fibrillar tau pathology. Some mutations are associated with inclusions in both neurons and glia (Fig. 2i), whereas others lead to a predominance of neuronal tau pathology. Depending on the mutation site, tau aggregates can be composed predominantly of $3 \mathrm{R}$ or $4 \mathrm{R}$ 
tau isoforms, or a mixture of both. MAPT mutations are located in the coding region or in the intron flanking the alternatively spliced exon 10 (Fig. 1b) and fall into two main functional categories: those that affect the alternative splicing of tau pre-mRNA, and those whose primary effect is at the protein level. The mechanisms by which the various MAPT mutations impair tau functions and generate filamentous tau inclusions are beyond the scope of this review, but they have recently been reviewed elsewhere (Refs 28, 66, 67).

FTLD-tau due to other gene abnormalities In addition to FTDP-17T, there remain many familial FTLD-tau cases for which no MAPT mutation has been discovered. Recently, an interesting association has been described between familial FTLD-tau and presenilin 1 (PS-1). Mutations in the genes encoding PS-1 and PS-2 (PSEN1 and PSEN2) account for the majority of early-onset familial AD (Ref. 68). However, PSEN1 mutations have also been identified in patients with an FTD presentation (Ref. 69). In one patient with familial FTD caused by the G183V PSEN1 mutation, the neuropathology was found to be consistent with $\mathrm{PiD}$ in the absence of $\beta$-amyloid deposits (Ref. 70), whereas another FTD family with the M146L mutation had both $\mathrm{PBs}$ and $\mathrm{AD}$ pathology (Ref. 71). However, some FTD patients with PSEN1 mutations have turned out to have typical AD pathology (Refs 72, 73). Finally, a family with FTD that was originally attributed to the PS-1 insR352 change was recently shown to have a GRN mutation and characteristic FTLDTDP pathology (Refs 74, 75). Currently, the mechanisms by which PSEN1 mutations contribute to neurodegeneration in a subset of FTD subjects are still elusive (Refs 76, 77).

\section{FTLD-TDP}

Until recently, most cases of tau-negative FTLD were thought to have no cellular inclusions and were classified as DLDH (Ref. 78). However, in the early 1990s, a novel pattern of pathology was first identified in patients with MND and dementia (MND-dementia), characterised by neuronal cytoplasmic inclusions (NCIs) and dystrophic neurites (DNs), in the superficial layers of frontotemporal neocortex and dentate granule cells of the hippocampus, that are immunoreactive for ubiquitin (ub-ir) but negative for tau and $\alpha$-synuclein (Refs 79, 80). Subsequently, similar ub-ir pathology was identified in a subgroup of patients with clinically pure FTD without motor symptoms (FTD-MND type or MND inclusion dementia) (Ref. 81). The pathology in cases of MNDdementia and FTD-MND type was found to be similar, regardless of the clinical phenotype (Ref. 82), and became known as FTLD with ubiquitinated inclusions (FTLD-U) (Ref. 83). With growing knowledge of this entity amongst neuropathologists and improvements in immunohistochemical techniques, FTLD-U became appreciated as the most common neuropathological subtype of FTLD (Refs 84, 85). The recognition of distinct patterns of FTLD-U pathology, raised the possibility that this might actually be a heterogeneous group of disorders, each with a different ubiquitinated protein (Refs 86, 87). However, this issue was largely resolved in 2006, when the transactive response (TAR) DNA-binding protein with a mass of $43 \mathrm{kDa}$ (TDP-43) was identified as the pathological protein in the vast majority of clinical and pathological FTLD-U subtypes as well as sporadic amyotrophic lateral sclerosis (ALS) (Refs 88, 89). The pathological group, now referred to as FTLDTDP (Ref. 22), includes sporadic cases and three autosomal dominant familial forms of FTLD (Table 1).

\section{Normal function of TDP-43}

TDP-43 is a protein comprising 414 amino acids, which is encoded by the TARDBP gene on chromosome 1 (Fig. 3a). It was first cloned as a human protein capable of binding to the transactive response DNA of human immunodeficiency virus Type 1 (Ref. 90), and later identified as part of a complex involved in splicing of the cystic fibrosis transmembrane conductance regulator gene (Ref. 91) and the apolipoprotein-A-II gene (Ref. 92). TDP-43 is highly conserved, ubiquitously expressed and predominantly localised to the nucleus. It consists of two RNA-recognition motifs and a glycine-rich C-terminal region (Fig. 3a). Recent studies have shown that TDP-43 continuously shuttles between the nucleus and cytoplasm - a process partially regulated by nuclear localisation signal (NLS) and nuclear export signal (NES) motifs (Refs 93, 94). In addition to its well-characterised role in regulation of 


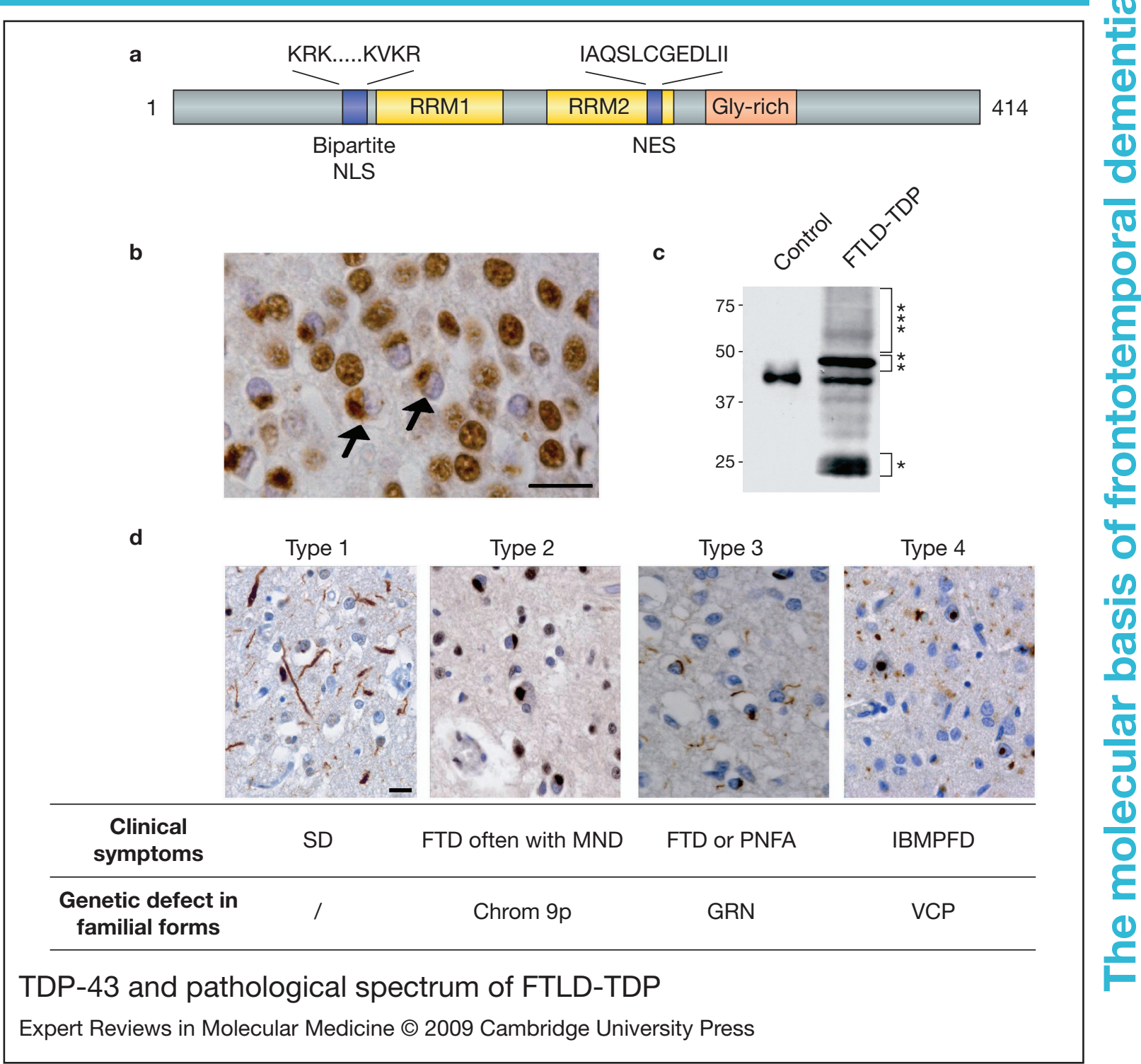

Figure 3. TDP-43 and pathological spectrum of FTLD-TDP. (a) Schematic diagram of TDP-43 with its characteristic functional domains. (b) TDP-43-positive cytoplasmic inclusions in the dentate granule cells. Note the absence of normal nuclear staining in cells with cytoplasmic inclusions (arrows). (c) Immunoblot analysis of urea fractions isolated from FTLD-TDP brain shows the highly characteristic biochemical signature with pathological bands at $25 \mathrm{kDa}\left({ }^{*}\right), 45 \mathrm{kDa}\left({ }^{* *}\right)$ and a high molecular mass smear $\left(^{(* *}\right)$, in addition to the normal TDP-43 band. (d) Based on morphological parameters, four FTLD-TDP subtypes can be delineated, each of which correlates with specific clinical phenotype and genetic abnormalities. Scale bars: $20 \mu \mathrm{m}$ (b, d). Abbreviations: RRM, RNA recognition motif; NLS, nuclear localisation sequence; NES, nuclear export sequence.

transcription and splicing, more recent studies suggest that TDP-43 is involved in other cellular processes such as micro RNA biogenesis, apoptosis, cell division, mRNA stabilisation and regulation of neuronal plasticity by acting as neuronal activity response factor (Refs 95, 96). The exon skipping and splicing inhibitory activity requires the C-terminal region of TDP-43, which interacts with other members of the heterogeneous nuclear ribonucleoprotein (hnRNP) family (Ref. 97). Finally, TDP-43 can also act as scaffold for nuclear bodies through interaction with survival motor neuron protein (Ref. 98). 
TDP-43 is the major pathological

\section{protein in FTLD-TDP}

As demonstrated in the initial reports and rapidly confirmed by numerous subsequent studies, antibodies against TDP-43 have proven to be the most sensitive and specific tool to detect all of the different types of the ub-ir pathology found in most cases of FTLD-U, including the NCIs, DNs and neuronal intranuclear inclusions (NIIs) (Fig. 3b, d) (Refs 88, 89, 99, 100). Moreover, the pathological forms of TDP-43 demonstrate evidence of abnormal processing, being hyperphosphorylated, ubiquitinated and Nterminally truncated (Ref. 88) (Fig. 3c). Of potential functional importance, the formation of TDP-43-positive inclusion bodies (either NCIs or NIIs) is consistently associated with a dramatic reduction of the normally diffuse nuclear staining (Ref. 88) (Fig. 3b), raising the possibility that essential nuclear functions of TDP-43 might be lost in FTLD-TDP.

The use of TDP-43 immunohistochemistry has clarified the spectrum of pathology in this FTLD subtype, by demonstrating previously unrecognised ubiquitin-negative pathology, including glial cytoplasmic inclusions (GCIs) in cells of presumed oligodendroglial lineage (Ref. 101), more diffuse neuronal cytoplasmic 'preinclusions' (Refs 102, 103) and delicate neurites in the CA1 region in a subset of FTLDTDP (Ref. 104). Antibodies raised against phosphorylated epitopes of TDP-43 (serine residues 379, 403, 404, 409 and 410) have further facilitated the neuropathological assessment, because they label only abnormal TDP-43 in inclusions, not the physiological nuclear TDP-43 (Refs 105, 106, 107).

\section{Pathological subtypes of FTLD-TDP}

FTLD-TDP pathology is heterogeneous with respect to the morphology and laminar distribution of pathological inclusions, leading to the description of four distinct pathological subtypes (Fig. 3d) (Refs 86, 87, 100). Using the classification scheme originally described by Sampathu and colleagues, FTLD-TDP Type 1 is characterised by an abundance of long neuritic profiles, predominantly in superficial cortical laminae, with few or no NCIs, NIIs or GCIs. Type 2 shows a predominance of NCIs in both superficial and deep cortical layers and frequent 'preinclusions' and GCIs. Neurites may be present but NIIs are rare or absent.
NCIs, which are typical of MND, are often present in lower motor neurons. Type 3 cases have abundant small neuritic profiles and NCIs, predominantly in the superficial cortical layers. Moderate numbers of lentiform NIIs can be found in affected cortical regions, especially in cases with a positive family history. The characteristic neuropathological feature of FTLD-TDP Type 4 pathology is an abundance of NIIs and DNs with only rare NCIs in cortical regions, and the absence of inclusions in the hippocampal dentate granule cells (Refs 108, 109). The relevance of this heterogeneity is supported by relatively specific correlations between the various patterns of pathology, the clinical phenotypes and the known genetic causes (Table 1, Fig. 3d, see below) (Refs 86, 99).

\section{Familial FTLD-TDP FTD due to GRN mutations}

Although many of the original FTDP-17 kindreds were found to have MAPT mutations (see section on FTLD-tau), there remained several families with FTD linked to the same region of chromosome 17 in which no MAPT mutation could be identified and some of these were known to have FTLD-U rather than tau pathology. This enigma was resolved in 2006, with the discovery of mutations in GRN, which is located just $1.7 \mathrm{Mb}$ centromeric to MAPT (Refs 14, 15). Progranulin (PGRN) is a multifunctional secreted protein that probably serves as a neurotrophic growth or survival factor, with functions in wound healing and inflammation (Refs 110, 111, 112). To date, 66 different pathogenic mutations have been described in 199 families (AD\&FTD Mutation database: http://www.molgen.ua.ac. be/FTDmutations), including all the remaining original FTDP-17 families. Mutations in GRN are at least as common a cause of familial FTD as MAPT (Refs 14, 15, 113, 114). Most pathogenic mutations are frameshift, splice site or nonsense mutations that produce premature stop codons, resulting in mutant mRNA transcripts that undergo nonsense-mediated decay (Refs 14, 15). The result of these null mutations is that the mutant protein is not produced, and there is a reduction of functional PGRN (haploinsufficiency). Consistent with this mechanism is the absence of PGRN in the ub-ir inclusions (Ref. 15). Instead, the pathology in all 
cases of FTD with GRN mutations is FTLD-TDP Type 3 with NIIs (Refs 88, 100, 115). A polymorphic variant in GRN located in the $3^{\prime}-$ untranslated region in a binding site for miR-659, has recently been shown to be a major risk factor for sporadic FTD, by suppressing the translation of GRN mRNA (Ref. 116).

\section{Chromosome-9p-linked FTD-MND}

Several groups have reported genetic linkage to chromosome 9p in families with a combination of FTD and MND (Refs 18, 19, 20). The identity of the abnormal gene is currently unknown. A sequence variation in the intraflagellar transport 74 gene (IFT74) was found to segregate with disease in one family, but no IFT74 mutations have been detected in other families with linkage to chromosome 9 (Ref. 117). Although only a small number of cases have been reported, the pathology is consistent with FTLD-TDP Type 2 combined with the characteristic features of MND (Ref. 100).

\section{FTD due to VCP mutations}

Mutations in VCP are the cause of a rare familial syndrome in which inclusion body myopathy, Paget disease of the bone and FTD (IMBPFD) show variable penetrance (Refs 16, 118). To date, 12 different missense mutations in VCP have been described in 29 families (AD\&FTD mutation database: http://www.molgen.ua.ac. be/FTDmutations). The first detailed study of the neuropathology in cases of IMBPFD with FTD demonstrated a unique and highly specific subtype of FTLD-U pathology, characterised by numerous NIIs and DNs, with very few NCIs (Refs 109, 119). This was subsequently shown to be a subtype of FTLD-TDP (Ref. 108) and was designated as Type 4 (Ref. 21).

VCP is a member of the AAA-ATPase gene superfamily, and functions as a molecular chaperone in a plethora of distinct cellular activities, including ubiquitin-dependent, endoplasmic-reticulum-associated protein degradation (ERAD), stress responses, programmed cell death, membrane fusion, nuclear envelope reconstruction and postmitotic Golgi reassembly. Many of these activities are directly or indirectly regulated by the UPS (Refs 118, 120). The mechanisms whereby VCP mutations cause neurodegeneration and TDP-43 accumulation are unclear, although it is tempting to speculate that alterations of ubiquitin-dependent protein degradation might have a role.

\section{Molecular mechanisms underlying FTLD-TDP}

The mechanistic aspects of abnormal TDP-43 processing and accumulation, the functional consequences of these abnormalities (loss-offunction versus toxic gain-of-function) as well as the links with GRN and VCP mutations are currently not well understood. The dramatic change in subcellular distribution of TDP-43 from the nucleus to the cytoplasm in inclusionbearing cells suggests that loss of pivotal nuclear TDP-43 functions in transcription and mRNA processing might have a pathogenic role. Consistent with this hypothesis, knockdown of TDP-43 in cell lines has been shown to have important consequences on essential metabolic processes, such as regulation of nuclear shape, cell cycle and apoptosis (Ref. 121). Moreover, TDP-43knockout mice are embryonic lethal (M. Neumann, unpublished). Alternatively, the generation and sequestration of abnormal TDP-43 species might have a toxic effect. Cortical inclusions in FTLD-TDP are selectively enriched for hyperphosphorylated C-terminal fragments (CTFs), compared with spinal cord inclusions, which contain more full-length TDP-43 (Refs 107, 122). This suggests that TDP-43 is differentially processed in different neuroanatomical regions and that TDP-43 CTFs might act as seeds for inclusion formation and aggregation, particularly in cortical neurons. Recently, one specific cleavage site (Arg208) was identified in the pathological TDP-43 CTFs purified from FTLD-TDP brains (Ref. 123). Overexpression of this CTF in cultured cells recapitulated some key features of FTLD-TDP, such as the formation of ubiquitinated and phosphorylated cytoplasmic aggregates; however, no clear effect on cell viability was observed (Ref. 123). An interesting link between TDP-43 and PGRN was recently reported by Zhang and co-workers who demonstrated caspase-dependent cleavage of TDP-43 upon PGRN knockdown in cultured cells (Ref. 124). Although it is widely accepted that TDP-43 can be cleaved by caspases, the influence of PGRN levels on this process was not replicated by another group (Ref. 125). Finally, an in vitro study showed that restricting TDP-43 from 
entering the nucleus by changing the NLS motif leads to the formation of cytoplasmic TDP-43 aggregates, with subsequent sequestration of endogenous TDP-43, resulting in a depletion of nuclear TDP-43 (Ref. 93). Thus, perturbation of the normal shuttling of TDP-43 between the nucleus and cytoplasm might predispose to both the formation of cytoplasmic inclusions and a loss of nuclear TDP-43.

Although initial studies failed to identify mutations in the TARDBP gene in FTLD-TDP (Refs 126, 127, 128), mutations were identified in familial and sporadic ALS, in early 2008 (Refs 129, 130). At least 30 different TARDBP mutations have now been identified, mostly in familial and sporadic ALS (Refs 131, 132, 133, $134,135,136,137,138,139,140)$. However, very recently, a TARDBP mutation (G295S) was reported in two unrelated patients with FTD and MND (Ref. 141). Except for the Y374X truncation mutation, all others are missense mutations, mostly in exon 6, which effect highly conserved amino residues in the C-terminal region of TDP-43. Elucidating the functional consequences of ALS-causing TARDBP mutations is likely to also provide important insights into the mechanism of neurodegeneration in FTLD-TDP. One current hypothesis is that TARDBP mutations might interfere with protein-protein interaction, thereby affecting intracellular transport or crucial transcriptional and splicing activities. Alternatively, the mutations might increase the generation of CTFs, promote the aggregation of TDP-43 and/or result in abnormal phosphorylation (Refs 129, 131, 135).

\section{FTLD-UPS}

Although initial data suggested that all FTLD-U cases are characterised by TDP- 43 pathology, recent follow-up studies have demonstrated that there are some cases in which the pathological inclusions are only detectable with markers of the ubiquitin proteasome system (FTLD-UPS), in which the pathological ubiquitinated protein(s) is still unknown (Refs 100, 104, 142, 143). The most common subtype of FTLD-UPS, representing $10-20 \%$ of all FTLD-U cases, was recently described in detail (Refs 144, 145). The highly unusual and consistent clinical and pathological features of these cases suggest that they represent a newly recognised, discrete entity and the term atypical
FTLD-U (aFTLD-U) was introduced. The clinical phenotype is sporadic, early-onset FTD with severe progressive behavioural and personality changes in the absence of aphasia or significant motor features. In addition to frontotemporal atrophy, severe degeneration of the anterior striatum and hippocampal sclerosis are common findings in aFTLD-U. TDP-43negative, ubiquitin-positive and p62-positive NCIs are most abundant in the frontal and temporal neocortex and hippocampus (Fig. 4a). However, the most intriguing pathological feature is the presence of NIIs in the dentate granule cells and cortical pyramidal neurons with a unique morphology, where they appear as elongated straight or curved bars or thick twisted filaments (Fig. 4b, c). The immunohistochemical profile of these NIIs is also unusual and different from the NCIs, because they are reactive for ubiquitin but not for p62 (Fig. 4d).

Familial FTD linked to chromosome 3 (FTD-3), due to mutations in CHMP2B (Ref. 17) has also recently been shown to have FTLD-UPS pathology (Ref. 146). So far, four families have been identified with different $C H M P 2 B$ mutations (AD\&FTD Mutation database: http:// www.molgen.ua.ac.be/FTDmutations). CHMP2B belongs to the chromatin-modifying protein/ charged multivesicular body protein family and is a component of ESCRT-III (endosomal sorting complex required for transport III), a complex involved in the degradation of surface receptor proteins and the formation of endocytotic multivesicular bodies. Although previous reports failed to detect abnormal protein deposits in brains of patients with FTD-3, a recent study demonstrated the presence of granular NCIs that were immunoreactive for ubiquitin and p62, but negative for TDP-43 and tau, predominantly in the dentate granule cells of the hippocampus (Fig. 4e) (Ref. 146).

\section{FTLD-IF}

Neuronal intermediate filament inclusion disease (NIFID) is an uncommon neurological disorder (Ref. 147) with pathology characterised by neuronal inclusions that are immunoreactive for all of the Class IV intermediate filaments (IFs) [light, medium and heavy neurofilament (NF) subunits and $\alpha$-internexin] (Refs 148, 149). The typical clinical presentation is early-onset sporadic FTD, associated with a pyramidal and/or extrapyramidal movement disorder 


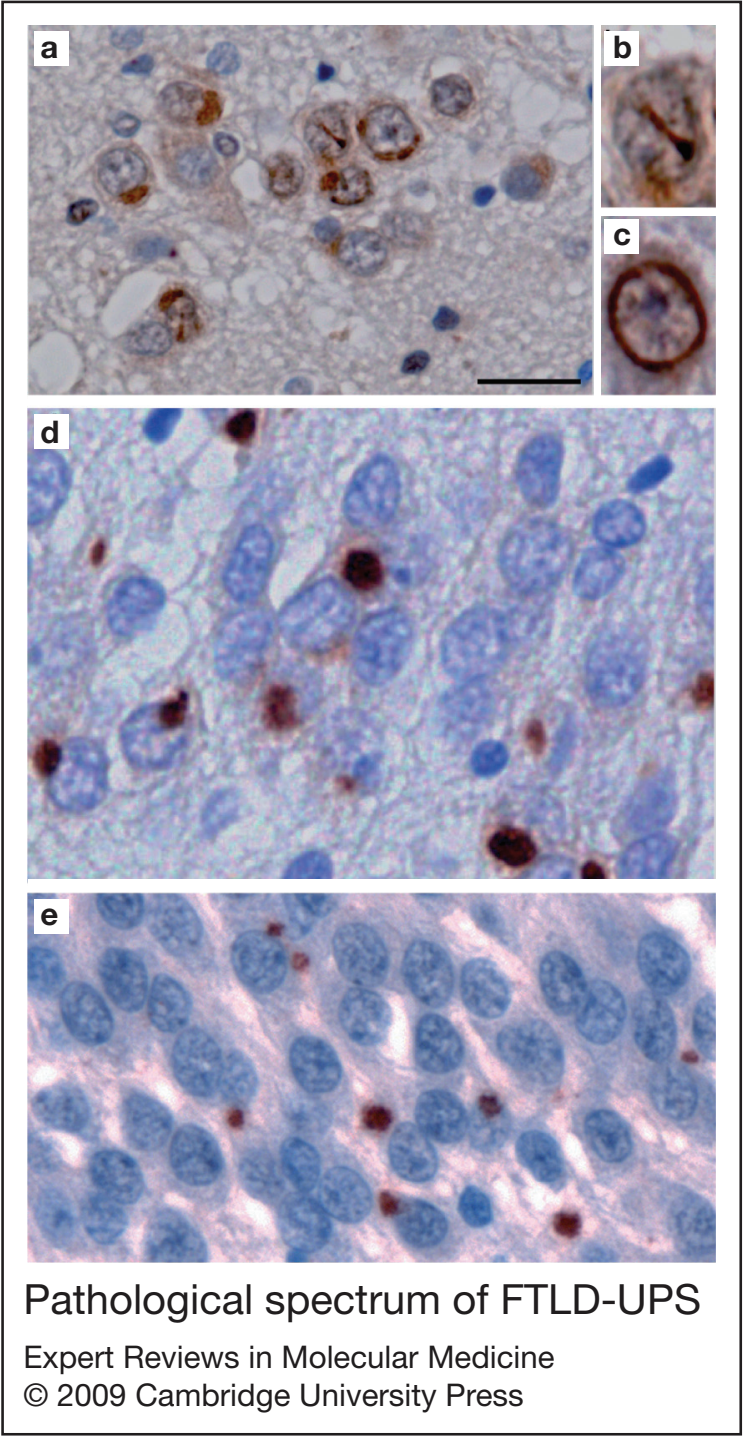

Figure 4. Pathological spectrum of FTLD-UPS. An aFTLD-U case with ubiquitin-immunoreactive cytoplasmic (a) and intranuclear inclusions (b, c) in dentate granule cells. (d) The cytoplasmic inclusions are also immunoreactive for p62 but the intranuclear inclusions are not. (e) Familial FTD caused by CHMP2B mutations (FTD-3) also have ubiquitin-positive cytoplasmic inclusions in the dentate granule cells. a-c, e, ubiquitin immunohistochemistry; d, p62 immunohistochemistry. Scale bar: $20 \mu \mathrm{m}(\mathrm{a}, \mathrm{d}, \mathrm{e})$; $10 \mu \mathrm{m}(\mathrm{b}, \mathrm{c})$.

(Refs 148, 150, 151, 152, 153, 154, 155, 156, 157). Additional clinical manifestations that have been reported include falls, dystonia, myoclonus, ophthalmoplegia, memory deficits, seizures, eating disorders and psychiatric symptoms. There have been two cases published with possible childhood onset (Refs 151, 153) and two with a questionable family history (Refs 152, 153).

The neuropathological findings in NIFID are heterogeneous, but have a number of consistent features (Refs 148, 150, 151, 152, 153, 154, 155, 156, 157). Chronic degenerative changes may affect a variety of cortical and subcortical regions, with the frontal and temporal lobes and caudate nucleus most consistently and severely involved. Several types of neuronal inclusions are found. The most common are small round Pick-body-like inclusions that are well-defined, slightly eosinophilic or basophilic and rarely argyrophilic (Fig. 5a). These are variably immunoreactive for ubiquitin, NF and $\alpha$-internexin but negative for tau, $\alpha$-synuclein and TDP-43 (Fig. 5b) (Ref. 149). The ultrastructure is a combination of electrondense granular material and dispersed short filaments (Refs 156, 158, 159). These are numerous in affected regions of cerebral neocortex, hippocampus and in select subcortical regions. Hyaline conglomerate inclusions are less frequent, and appear as irregular, multilobulated masses that often compress the nucleus (Fig. 5c). They are weakly eosinophilic, with a glassy, filamentous appearance and sometimes have a dense, brightly eosinophilic core. These stain intensely with the Bielschowsky silver method and are more consistently and intensely immunoreactive for IFs (Fig. 5d). The ultrastructure is compact, interlacing bundles of filaments (Ref. 158). NCI with other morphologies may also be present, and there are often IF-immunoreactive swollen axons. A less consistent feature is the presence of eosinophilic NIIs that are intensely immunoreactive for ubiquitin but generally not reactive for IFs (Fig. 5e) (Refs 149, 153, 154, 158).

Neuronal IFs include the triplet of light (68 $\mathrm{kDa})$, medium (145 kDa) and heavy (200 kDa) NF subunits, $\alpha$-internexin and peripherin (Ref. 160). Based on sequence homology, the NF proteins and $\alpha$-internexin have been grouped together as Class IV IFs (Ref. 161). The expression of $\alpha$-internexin precedes that of NFs during development and, in the adult brain, $\alpha$-internexin is expressed at lower levels and in more restricted neuronal populations (Ref. 162). The Class IV IFs can coassemble (Ref. 163), and 


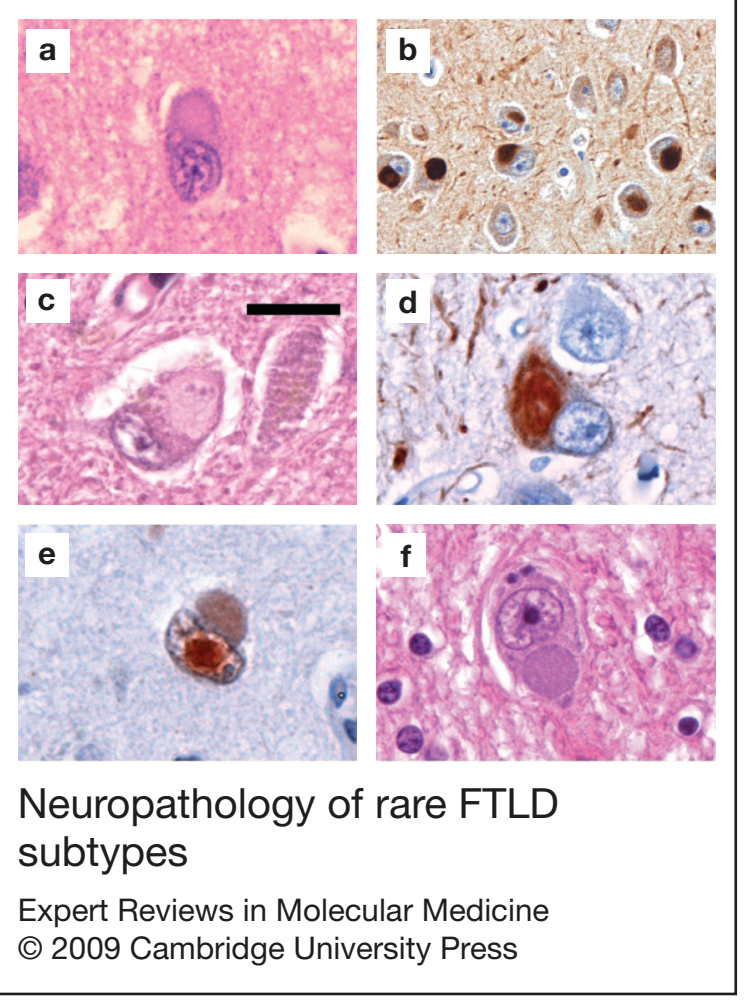

Figure 5. Neuropathology of rare FTLD subtypes. (a-d) Neuronal intermediate filament inclusion disease. The most common type of inclusion is Pick-body-like inclusions that are visible with haematoxylin and eosin stain (a) and immunoreactive for $\alpha$-internexin (b). Hyaline conglomerate inclusions are also present (c) and are immunoreactive for phosphorylated neurofilament (d). (e) Neuronal intranuclear inclusions that are present in some cases of FTLDIF are strongly immunoreactive for ubiquitin but do not stain for intermediate filaments. Note the presence of a Pick-body-like inclusion in the cytoplasm of the same neuron. (f) Typical inclusion of basophilic inclusion body disease. Scale bar: $20 \mu \mathrm{m}$ (a, c-f); $40 \mu \mathrm{m}$ (b).

are part of the normal neuronal cytoskeleton, that is involved in maintaining cell structure and intracellular transport (Ref. 164). The degree and pattern of phosphorylation of NFs is essential for normal intracellular localisation and function (Ref. 160). The inclusions in NIFID are variably reactive with antibodies against phosphorylated and phosphorylation-independent epitopes of all three NF subunits; however, $\alpha$ internexin immunohistochemistry is the most sensitive (Refs 148, 149). Although NF can be a minor component of the characteristic inclusion bodies of many different neurodegenerative conditions (including AD, PD, DLB and MND) (Ref. 160), $\alpha$-internexin immunoreactivity is relatively specific for NIFID (Ref. 165). Because the abnormal protein aggregates found in NIFID are immunoreactive for IFs but not for any other proteins currently known to be associated with neurodegenerative disease, the designation of NIFID or FTLD-IF is appropriate. However, the fact that IF proteins show no abnormal molecular modification in NIFID (Refs 149, 158, 165) and no pathogenic variants of the corresponding genes have been identified (Ref. 166), leaves open the possibility that some other protein might have a more central role in the pathogenesis of this condition (Refs 150,153).

\section{BIBD}

BIBDis a term that has been used for a small number of clinically and pathologically heterogeneous cases, in which the common finding is neuronal cytoplasmic inclusions that are basophilic with haematoxylin and eosin stain (Fig. 5f). The clinical phenotypes include sporadic ALS (Ref. 167), familial ALS (Ref. 168), ALS with dementia (Refs 150, 169, 170) and pure FTD (Refs 150, 171). Although cases of BIBD with clinical FTD show chronic degeneration of the frontotemporal neocortex, the inclusions tend to be most numerous in subcortical regions, such as the basal ganglia and brainstem tegmentum (Refs 150, 171). The inclusions are round, oval or crescentic, weakly argyrophilic and can be detected with histochemical stains for RNA (such as cresyl violet, methyl green pyronine and acridine orange). Immunohistochemical studies have found them to be negative for tau, $\alpha$ synuclein, NF, $\alpha$-internexin, tubulin, actin and TDP-43 (Refs 100, 150, 169, 171). Reports of ubiquitin immunoreactivity are inconsistent but some are positive for p62 (Ref. 150). The biochemical composition of the signature inclusions in BIBD is not presently known.

\section{FTLD-ni}

Until recently, most cases of tau-negative FTLD were thought to have no cellular inclusions and were classified as DLDH (Ref. 78). However, with the use of sensitive immunohistochemical techniques, most of these cases are found to have ubiquitin-immunoreactive pathology (previously designated FTLD-U) (Ref. 85) that is usually also reactive for TDP-43 (Ref. 99). 
Cases of true DLDH are now considered to be uncommon, and the existence of DLDH as a distinct entity has been questioned. The terminology that is now recommended for rare cases of FTLD in which no inclusions can be demonstrated with special histochemical stains or immunohistochemistry is FTLD-ni (no inclusions) (Ref. 22).

\section{Other pathological causes of FTD}

There are a number of rare neurodegenerative conditions, with unknown biochemical defects, that can present as FTD, such as hereditary diffuse leukoencephalopathy with spheroids (Ref. 172) and neuronal intranuclear inclusion disease (Ref. 173). In addition, most of the common neurodegenerative causes of dementia can occasionally fulfill clinical diagnostic criteria for FTD. Although the frequency of these 'frontal variants' and their overall contribution to FTD is difficult to determine, some studies have found more than $20 \%$ of clinical FTD to be associated with the pathology of $\mathrm{AD}, \mathrm{DLB}, \mathrm{CJD}$ or vascular disease (Refs 174, 175). The frontal variant of $\mathrm{AD}$ is best recognised and could account for $17 \%$ of all FTD (Refs 174, 175). Attempts to demonstrate a correlation between the regional severity of the AD pathology and the clinical phenotype have been inconsistent (Refs 56, 57, 176). One recent study has described additional glial tau pathology in cases of AD with clinical PPA, suggesting a pathological overlap with FTLDtau (Ref. 177). Another recent finding of interest is the presence of TDP-43 pathology in a significant proportion of AD and DLB cases (Refs 178, 179, 180, 181, 182). Although this often has a restricted anatomical distribution, it is sometimes extensive and might closely resemble FTLD-TDP (Ref. 181). Whether or not this additional pathology alters the clinical phenotype is yet to be determined.

\section{Outstanding research questions}

Despite the enormous gains made over the past few years in our understanding of the molecular basis of FTD, many fundamental issues are still unresolved. The FTLD gene on chromosome 9 remains to be identified and there is probably at least one other genetic cause of familial FTLDtau. The normal functions of PGRN and TDP-43 in the nervous system need to be clarified, as do the mechanisms by which mutations in GRN,
$V C P$ and TARDBP lead to TDP-43 pathology. The possible role of GRN and TARDBP genetic variants in other (non-FTLD) neurodegenerative disease requires further investigation. The development of new animal models, particularly of FTLD-TDP, should help to answer these questions and will be crucial in the development of targeted therapies. Efforts to develop in vivo biomarkers that distinguish different subgroups of FTLD and aid in diagnosis and monitoring of disease are still preliminary (Refs 183, 184, 185, 186). Despite the enormous amount of work that lies ahead, recent discoveries have greatly improved our ability to offer meaningful genetic counselling for FTLD families and bring us much closer to developing useful diagnostic tests and rational therapies.

\section{Acknowledgements and funding}

This work was supported by funding from Stavros-Niarchos Foundation and the Synapsis Foundation (M.N.), the Swiss National Science Foundation (M.T.), the Canadian Institutes for Health Research and the Pacific Alzheimer Research Foundation (I.R.A.M.). The authors would like to thank the peer reviewers of this work for their constructive comments.

\section{References}

1 McKhann, G.M. et al. (2001) Clinical and pathological diagnosis of frontotemporal dementia: report of the Work Group on Frontotemporal Dementia and Pick's Disease. Archives of Neurology 58, 1803-1809

2 Neary, D. et al. (1998) Frontotemporal lobar degeneration: a consensus on clinical diagnostic criteria. Neurology 51, 1546-1554

3 The Lund and Manchester Groups (1994) Clinical and neuropathological criteria for frontotemporal dementia. Journal of Neurology Neurosurgery and Psychiatry 57, 416-418

4 Padovani, A. et al. (2007) Extrapyramidal symptoms in Frontotemporal Dementia: prevalence and clinical correlations. Neuroscience Letters 422, 39-42

5 Lomen-Hoerth, C., Anderson, T. and Miller, B. (2002) The overlap of amyotrophic lateral sclerosis and frontotemporal dementia. Neurology 59, 1077-1079

6 Feldman, H. et al. (2003) A Canadian cohort study of cognitive impairment and related dementias (ACCORD): study methods and baseline results. Neuroepidemiology 22, 265-274 
7 Bird, T. et al. (2003) Epidemiology and genetics of frontotemporal dementia/Pick's disease. Annals of Neurology 54 Suppl. 5, S29-31

8 Stevens, M. et al. (1998) Familial aggregation in frontotemporal dementia. Neurology 50, 1541-1545

9 Rosso, S.M. et al. (2003) Frontotemporal dementia in The Netherlands: patient characteristics and prevalence estimates from a population-based study. Brain 126, 2016-2022

10 Chow, T.W. etal. (1999) Inheritance of frontotemporal dementia. Archives of Neurology 56, 817-822

11 Hutton, M. et al. (1998) Association of missense and 5 -splice-site mutations in tau with the inherited dementia FTDP-17. Nature 393, 702-705

12 Poorkaj, P. et al. (1998) Tau is a candidate gene for chromosome 17 frontotemporal dementia. Annals of Neurology 43, 815-825

13 Spillantini, M.G. et al. (1998) Mutation in the tau gene in familial multiple system tauopathy with presenile dementia. Proceedings of the National Academy of Sciences of the United States of America 95, 7737-7741

14 Cruts, M. et al. (2006) Null mutations in progranulin cause ubiquitin-positive frontotemporal dementia linked to chromosome 17q21. Nature 442, 920-924

15 Baker, M. et al. (2006) Mutations in progranulin cause tau-negative frontotemporal dementia linked to chromosome 17. Nature 442, 916-919

16 Watts, G.D. et al. (2004) Inclusion body myopathy associated with Paget disease of bone and frontotemporal dementia is caused by mutant valosin-containing protein. Nature Genetics 36, 377-381

17 Skibinski, G. et al. (2005) Mutations in the endosomal ESCRTIII-complex subunit CHMP2B in frontotemporal dementia. Nature Genetics 37, 806-808

18 Valdmanis, P.N. et al. (2007) Three families with amyotrophic lateral sclerosis and frontotemporal dementia with evidence of linkage to chromosome 9p. Archives of Neurology 64, 240-245

19 Morita, M. et al. (2006) A locus on chromosome 9p confers susceptibility to ALS and frontotemporal dementia. Neurology 66, 839-844

20 Vance, C. et al. (2006) Familial amyotrophic lateral sclerosis with frontotemporal dementia is linked to a locus on chromosome 9p13.2-21.3. Brain 129, 868-876

21 Cairns, N.J. et al. (2007) Neuropathologic diagnostic and nosologic criteria for frontotemporal lobar degeneration: consensus of the Consortium for Frontotemporal Lobar Degeneration. Acta Neuropathologica 114, 5-22

22 Mackenzie, I.R. et al. (2009) Nomenclature for neuropathologic subtypes of frontotemporal lobar degeneration: consensus recommendations. Acta Neuropathologica 117, 15-18

23 Cleveland, D.W., Hwo, S.Y. and Kirschner, M.W. (1977) Purification of tau, a microtubule-associated protein that induces assembly of microtubules from purified tubulin. Journal of Molecular Biology 116, 207-225

24 Weingarten, M.D. et al. (1975) A protein factor essential for microtubule assembly. Proceedings of the National Academy of Sciences of the United States of America 72, 1858-1862

25 Baker, M. et al. (1999) Association of an extended haplotype in the tau gene with progressive supranuclear palsy. Human Molecular Genetics $8,711-715$

26 Stefansson, H. et al. (2005) A common inversion under selection in Europeans. Nature Genetics 37, 129-137

27 Andreadis, A., Brown, W.M. and Kosik, K.S. (1992) Structure and novel exons of the human tau gene. Biochemistry 31, 10626-10633

28 Goedert, M. and Spillantini, M.G. (2006) A century of Alzheimer's disease. Science 314, 777-781

29 Goedert, M. et al. (1989) Multiple isoforms of human microtubule-associated protein tau: sequences and localization in neurofibrillary tangles of Alzheimer's disease. Neuron 3, 519-526

30 Tolnay, M. and Probst, A. (2003) The neuropathological spectrum of neurodegenerative tauopathies. IUBMB Life 55, 299-305

31 Lee, V.M., Goedert, M. and Trojanowski, J.Q. (2001) Neurodegenerative tauopathies. Annual Review of Neuroscience 24, 1121-1159

32 Delacourte, A. (2008) Tau, a biological marker of neurodegenerative diseases. Handbook of Clinical Neurology 89, 161-172

33 Onari, K. and Spatz, H. (1926) Anatomische Beiträge zur Lehre von der Pickschen umschriebenen Großhirnrinden-Atrophie ("Picksche Krankheit"). Zeitschrift für die gesamte Neurologie und Psychiatrie 101, 470-511

34 Alzheimer, A. (1911) Über eigenartige Krankheitsfälle des späteren Alters. Zeitschrift für die gesamte Neurologie und Psychiatrie 4, 356-385

35 Pick, A. (1892) Ueber die Beziehungen der senilen Hirnatrophie zur Aphasie. Prager medizinische Wochenschrift 17, 165-167 
36 Zhukareva, V. et al. (2002) Sporadic Pick's disease: a tauopathy characterized by a spectrum of pathological tau isoforms in gray and white matter. Annals of Neurology 51, 730-739

37 Mott, R.T. et al. (2005) Neuropathologic, biochemical, and molecular characterization of the frontotemporal dementias. Journal of Neuropathology and Experimental Neurology $64,420-428$

38 Steele, J., Richardson, J. and Olszewski, J. (1964) Progressive supranuclear palsy; a heterogeneous degeneration involving the brain stem, basal ganglia and cerebellum with vertical gaze and pseudobulbar palsy, nuclear dystonia and dementia. Archives of Neurology 10, 333-359

39 Sha, S. et al. (2006) Are frontotemporal lobar degeneration, progressive supranuclear palsy and corticobasal degeneration distinct diseases? Nature Clinical Practice. Neurology 2, 658-665

40 Bigio, E.H., Brown, D.F. and White, C.L. 3rd (1999) Progressive supranuclear palsy with dementia: cortical pathology. Journal of Neuropathology and Experimental Neurology 58, 359-364

41 Dickson, D.W., Rademakers, R. and Hutton, M.L. (2007) Progressive supranuclear palsy: pathology and genetics. Brain Pathology 17, 74-82

42 Pittman, A.M. et al. (2005) Linkage disequilibrium fine mapping and haplotype association analysis of the tau gene in progressive supranuclear palsy and corticobasal degeneration. Journal of Medical Genetics 42, 837-846

43 Di Maria, E. et al. (2000) Corticobasal degeneration shares a common genetic background with progressive supranuclear palsy. Annals of Neurology 47, 374-377

44 Rebeiz, J.J., Kolodny, E.H. and Richardson, E.P. Jr. (1968) Corticodentatonigral degeneration with neuronal achromasia. Archives of Neurology 18, 20-33

45 Grimes, D.A., Lang, A.E. and Bergeron, C.B. (1999) Dementia as the most common presentation of cortical-basal ganglionic degeneration. Neurology 53, 1969-1974

46 Feany, M.B. and Dickson, D.W. (1995) Widespread cytoskeletal pathology characterizes corticobasal degeneration. American Journal of Pathology 146, 1388-1396

47 Tolnay, M. and Probst, A. (2008) Argyrophilic grain disease. Handbook of Clinical Neurology 89, 553-563

48 Braak, H. and Braak, E. (1989) Cortical and subcortical argyrophilic grains characterize a disease associated with adult onset dementia.
Neuropathology and Applied Neurobiology $15,13-26$

49 Steuerwald, G.M. et al. (2007) Clinical characteristics of dementia associated with argyrophilic grain disease. Dementia and Geriatric Cognitive Disorders 24, 229-234

50 Togo, T. et al. (2005) Clinical features of argyrophilic grain disease: a retrospective survey of cases with neuropsychiatric symptoms. American Journal of Geriatric Psychiatry 13, 1083-1091

51 Ishihara, K. et al. (2005) Argyrophilic grain disease presenting with frontotemporal dementia: a neuropsychological and pathological study of an autopsied case with presenile onset.

Neuropathology 25, 165-170

52 Tsuchiya, K. et al. (2001) Argyrophilic grain disease mimicking temporal Pick's disease: a clinical, radiological, and pathological study of an autopsy case with a clinical course of 15 years. Acta Neuropathologica 102, 195-199

53 Zhukareva, V. et al. (2002) Biochemical analysis of tau proteins in argyrophilic grain disease, Alzheimer's disease, and Pick's disease: a comparative study. American Journal of Pathology 161, 1135-1141

54 Togo, T. et al. (2002) Argyrophilic grain disease is a sporadic 4-repeat tauopathy. Journal of Neuropathology and Experimental Neurology 61, 547-556

55 Tolnay, M. et al. (2002) Argyrophilic grain disease and Alzheimer's disease are distinguished by their different distribution of tau protein isoforms. Acta Neuropathologica 104, 425-434

56 Johnson, J.K. et al. (1999) Clinical and pathological evidence for a frontal variant of Alzheimer disease. Archives of Neurology 56, 1233-1239

57 Taylor, K.I. et al. (2008) Clinical course of neuropathologically confirmed frontal-variant Alzheimer's disease. Nature Clinical Practice. Neurology 4, 226-232

58 Jellinger, K.A. and Attems, J. (2007) Neurofibrillary tangle-predominant dementia: comparison with classical Alzheimer disease. Acta Neuropathologica 113, 107-117

59 Bigio, E.H. et al. (2001) Frontal lobe dementia with novel tauopathy: sporadic multiple system tauopathy with dementia. Journal of Neuropathology and Experimental Neurology 60, 328-341

60 Kovacs, G.G. et al. (2008) White matter tauopathy with globular glial inclusions: a distinct sporadic 
frontotemporal lobar degeneration. Journal of Neuropathology and Experimental Neurology 67, 963-975

61 Dickson, D.W. et al. (1994) Hippocampal sclerosis: a common pathological feature of dementia in very old ( $>$ or $=80$ years of age) humans. Acta Neuropathologica 88, 212-221

62 Beach, T.G. et al. (2003) Hippocampal sclerosis dementia with tauopathy. Brain Pathology 13, 263-278

63 Probst, A., Taylor, K.I. and Tolnay, M. (2007) Hippocampal sclerosis dementia: a reappraisal. Acta Neuropathologica 114, 335-345

64 Wilhelmsen, K.C. et al. (1994) Localization of disinhibition-dementia-parkinsonismamyotrophy complex to 17q21-22. American Journal of Human Genetics 55, 1159-1165

65 Foster, N.L. et al. (1997) Frontotemporal dementia and parkinsonism linked to chromosome 17: a consensus conference. Annals of Neurology 41, 706-715

66 van Swieten, J. and Spillantini, M.G. (2007) Hereditary frontotemporal dementia caused by Tau gene mutations. Brain Pathology 17, 63-73

67 Goedert, M. (2005) Tau gene mutations and their effects. Movement Disorders 20 Suppl 12, S45-52

68 Roberson, E.D. and Mucke, L. (2006) 100 years and counting: prospects for defeating Alzheimer's disease. Science 314, 781-784

69 van der Zee, J., Sleegers, K. and Van Broeckhoven, C. (2008) Invited article: the Alzheimer diseasefrontotemporal lobar degeneration spectrum. Neurology 71, 1191-1197

70 Dermaut, B. et al. (2004) A novel presenilin 1 mutation associated with Pick's disease but not beta-amyloid plaques. Annals of Neurology 55, 617-626

71 Halliday, G.M. et al. (2005) Pick bodies in a family with presenilin-1 Alzheimer's disease. Annals of Neurology 57, 139-143

72 Zekanowski, C. et al. (2006) Two novel presenilin 1 gene mutations connected with frontotemporal dementia-like clinical phenotype: genetic and bioinformatic assessment. Experimental Neurology 200, 82-88

73 Rippon, G.A. et al. (2003) Presenilin 1 mutation in an african american family presenting with atypical Alzheimer dementia. Archives of Neurology 60, 884-888

74 Boeve, B.F. et al. (2006) Frontotemporal dementia and parkinsonism associated with the IVS1 + 1G$>$ A mutation in progranulin: a clinicopathologic study. Brain 129, 3103-3114
75 Pickering-Brown, S.M. et al. (2006) Mutations in progranulin explain atypical phenotypes with variants in MAPT. Brain 129, 3124-3126

76 Kumar-Singh, S. and Van Broeckhoven, C. (2007) Frontotemporal lobar degeneration: current concepts in the light of recent advances. Brain Pathology 17, 104-114

77 Hutton, M. (2004) Presenilin mutations associated with fronto-temporal dementia. Annals of Neurology 55, 604-606

78 Knopman, D.S. et al. (1990) Dementia lacking distinctive histologic features: a common nonAlzheimer degenerative dementia. Neurology 40, 251-256

79 Wightman, G. et al. (1992) Hippocampal and neocortical ubiquitin-immunoreactive inclusions in amyotrophic lateral sclerosis with dementia. Neuroscience Letters 139, 269-274

80 Okamoto, K. et al. (1992) Ubiquitin-positive intraneuronal inclusions in the extramotor cortices of presenile dementia patients with motor neuron disease. Journal of Neurology 239, 426-430

81 Jackson, M., Lennox, G. and Lowe, J. (1996) Motor neurone disease-inclusion dementia.

Neurodegeneration 5, 339-350

82 Mackenzie, I.R. and Feldman, H.H. (2005) Ubiquitin immunohistochemistry suggests classic motor neuron disease, motor neuron disease with dementia, and frontotemporal dementia of the motor neuron disease type represent a clinicopathologic spectrum. Journal of Neuropathology and Experimental Neurology 64, 730-739

83 Lowe, J. and Rossor, M.N. (2003) Frontotemporal lobar degeneration. In Neurodegeneration: the molecular pathology of dementia and movement disorders (Dickson D.W., eds), pp. 342-348, ISN Neuropath Press, Basel

84 Lipton, A.M., White, C.L. 3rd and Bigio, E.H. (2004) Frontotemporal lobar degeneration with motor neuron disease-type inclusions predominates in 76 cases of frontotemporal degeneration. Acta Neuropathologica 108, 379-385

85 Mackenzie, I.R. et al. (2006) Dementia lacking distinctive histology (DLDH) revisited. Acta Neuropathologica 112, 551-559

86 Mackenzie, I.R. et al. (2006) Heterogeneity of ubiquitin pathology in frontotemporal lobar degeneration: classification and relation to clinical phenotype. Acta Neuropathologica 112, 539-549

87 Sampathu, D.M. et al. (2006) Pathological heterogeneity of frontotemporal lobar degeneration with ubiquitin-positive inclusions 
delineated by ubiquitin immunohistochemistry and novel monoclonal antibodies. American Journal of Pathology 169, 1343-1352

88 Neumann, M. et al. (2006) Ubiquitinated TDP-43 in frontotemporal lobar degeneration and amyotrophic lateral sclerosis. Science 314, 130-133

89 Arai, T. et al. (2006) TDP-43 is a component of ubiquitin-positive tau-negative inclusions in frontotemporal lobar degeneration and amyotrophic lateral sclerosis. Biochemical and Biophysical Research Communications 351, 602-611

$90 \mathrm{Ou}$, S.H. et al. (1995) Cloning and characterization of a novel cellular protein, TDP-43, that binds to human immunodeficiency virus type 1 TAR DNA sequence motifs. Journal of Virology 69, 3584-3596

91 Buratti, E. et al. (2001) Nuclear factor TDP-43 and SR proteins promote in vitro and in vivo CFTR exon 9 skipping. EMBO Journal 20, 1774-1784

92 Mercado, P.A. et al. (2005) Depletion of TDP 43 overrides the need for exonic and intronic splicing enhancers in the human apoA-II gene. Nucleic Acids Research 33, 6000-6010

93 Winton, M.J. et al. (2008) Disturbance of nuclear and cytoplasmic TAR DNA-binding protein (TDP-43) induces disease-like redistribution, sequestration, and aggregate formation. Journal of Biological Chemistry 283, 13302-13309

94 Ayala, Y.M. et al. (2008) Structural determinants of the cellular localization and shuttling of TDP-43. Journal of Cell Science 121, 3778-3785

95 Strong, M.J. et al. (2007) TDP43 is a human low molecular weight neurofilament (hNFL) mRNAbinding protein. Molecular and Cellular Neurosciences 35, 320-327

96 Wang, I.F. et al. (2008) TDP-43, the signature protein of FTLD-U, is a neuronal activityresponsive factor. Journal of Neurochemistry $105,797-806$

97 Buratti, E. et al. (2005) TDP-43 binds heterogeneous nuclear ribonucleoprotein A/B through its Cterminal tail: an important region for the inhibition of cystic fibrosis transmembrane conductance regulator exon 9 splicing. Journal of Biological Chemistry 280, 37572-37584

98 Wang, I.F., Reddy, N.M. and Shen, C.K. (2002) Higher order arrangement of the eukaryotic nuclear bodies. Proceedings of the National Academy of Sciences of the United States of America 99, 13583-13588
99 Davidson, Y. et al. (2007) Ubiquitinated pathological lesions in frontotemporal lobar degeneration contain the TAR DNA-binding protein, TDP-43. Acta Neuropathologica 113, 521-533

100 Cairns, N.J. et al. (2007) TDP-43 in familial and sporadic frontotemporal lobar degeneration with ubiquitin inclusions. American Journal of Pathology 171, 227-240

101 Neumann, M. et al. (2007) TDP-43-positive white matter pathology in frontotemporal lobar degeneration with ubiquitin-positive inclusions. Journal of Neuropathology and Experimental Neurology 66, 177-183

102 Brandmeir, N.J. et al. (2008) Severe subcortical TDP-43 pathology in sporadic frontotemporal lobar degeneration with motor neuron disease. Acta Neuropathologica 115, 123-131

103 Geser, F. et al. (2009) Clinical and pathological continuum of multisystem TDP-43

proteinopathies. Archives of Neurology 66, 180-189

104 Hatanpaa, K.J. et al. (2008) TAR DNA-Binding Protein 43 Immunohistochemistry Reveals Extensive Neuritic Pathology in FTLD-U: A Midwest-Southwest Consortium for FTLD Study. Journal of Neuropathology and Experimental Neurology 67, 271-279

105 Hasegawa, M. et al. (2008) Phosphorylated TDP-43 in frontotemporal lobar degeneration and amyotrophic lateral sclerosis. Annals of Neurology 64, 60-70

106 Inukai, Y. et al. (2008) Abnormal phosphorylation of Ser409/410 of TDP-43 in FTLD-U and ALS. FEBS Letters 582, 2899-2904

107 Neumann, M. et al. (2009) Phosphorylation of S409/410 of TDP-43 is a consistent feature in all sporadic and familial forms of TDP-43 proteinopathies. Acta Neuropathologica 117, 137-149

108 Neumann, M. et al. (2007) TDP-43 in the ubiquitin pathology of frontotemporal dementia with VCP gene mutations. Journal of Neuropathology and Experimental Neurology 66, 152-157

109 Forman, M.S. et al. (2006) Novel ubiquitin neuropathology in frontotemporal dementia with valosin-containing protein gene mutations. Journal of Neuropathology and Experimental Neurology 65, 571-581

$110 \mathrm{He}, \mathrm{Z}$. and Bateman, A. (2003) Progranulin (granulin-epithelin precursor, PC-cell-derived growth factor, acrogranin) mediates tissue repair and tumorigenesis. Journal of Molecular Medicine 1, 600-612 
111 Van Damme, P. et al. (2008) Progranulin functions as a neurotrophic factor to regulate neurite outgrowth and enhance neuronal survival. Journal of Cell Biology 181, 37-41

$112 \mathrm{He}$, Z. et al. (2003) Progranulin is a mediator of the wound response. Nature Medicine 9, 225-229

113 Pickering-Brown, S.M. et al. (2008) Frequency and clinical characteristics of progranulin mutation carriers in the Manchester frontotemporal lobar degeneration cohort: comparison with patients with MAPT and no known mutations. Brain 131, 721-731

114 Gass, J. et al. (2006) Mutations in progranulin are a major cause of ubiquitin-positive frontotemporal lobar degeneration. Human Molecular Genetics 15, 2988-3001

115 Mackenzie, I.R. (2007) The neuropathology and clinical phenotype of FTD with progranulin mutations. Acta Neuropathologica 114, 49-54

116 Rademakers, R. et al. (2008) Common variation in the miR-659 binding-site of GRN is a major risk factor for TDP43-positive frontotemporal dementia. Human Molecular Genetics 17, 3631-3642

117 Momeni, P. et al. (2006) Analysis of IFT74 as a candidate gene for chromosome 9p-linked ALS-FTD. BMC Neurology 6, 44

118 Kimonis, V.E. et al. (2008) VCP disease associated with myopathy, Paget disease of bone and frontotemporal dementia: review of a unique disorder. Biochimica et Biophysica Acta 1782, 744-748

119 Schroder, R. et al. (2005) Mutant valosin-containing protein causes a novel type of frontotemporal dementia. Annals of Neurology 57, 457-461

120 Guinto, J.B. et al. (2007) Valosin-containing protein and the pathogenesis of frontotemporal dementia associated with inclusion body myopathy. Acta Neuropathologica 114, 55-61

121 Ayala, Y.M., Misteli, T. and Baralle, F.E. (2008) TDP-43 regulates retinoblastoma protein phosphorylation through the repression of cyclindependent kinase 6 expression. Proceedings of the National Academy of Sciences of the United States of America 105, 3785-3789

122 Igaz, L.M. et al. (2008) Enrichment of C-terminal fragments in TAR DNA-binding protein-43 cytoplasmic inclusions in brain but not in spinal cord of frontotemporal lobar degeneration and amyotrophic lateral sclerosis. American Journal of Pathology 173, 182-194

123 Igaz, L.M. et al. (2009) Expression Of TDP-43 C-terminal fragments in vitro recapitulates pathological features of TDP-43 proteinopathies. Journal of Biological Chemistry 284, 8516-8524

124 Zhang, Y.J. et al. (2007) Progranulin mediates caspase-dependent cleavage of TAR DNA binding protein-43. Journal of Neuroscience 27, 10530-10534

125 Shankaran, S.S. et al. (2008) Missense mutations in the progranulin gene linked to frontotemporal lobar degeneration with ubiquitinimmunoreactive inclusions reduce progranulin production and secretion. Journal of Biological Chemistry 283, 1744-1753

126 Gijselinck, I. et al. (2009) Neuronal inclusion protein TDP-43 has no primary genetic role in FTD and ALS. Neurobiology of Aging 30, 1329-1331

127 Schumacher, A. et al. (2009) No association of TDP-43 with sporadic frontotemporal dementia. Neurobiology of Aging 30, 157-159

128 Rollinson, S. et al. (2007) TDP-43 gene analysis in frontotemporal lobar degeneration. Neuroscience Letters 419, 1-4

129 Kabashi, E. et al. (2008) TARDBP mutations in individuals with sporadic and familial amyotrophic lateral sclerosis. Nature Genetics $40,572-574$

130 Sreedharan, J. et al. (2008) TDP-43 mutations in familial and sporadic amyotrophic lateral sclerosis. Science 319, 1668-1672

131 Rutherford, N.J. et al. (2008) Novel mutations in TARDBP (TDP-43) in patients with familial amyotrophic lateral sclerosis. PLoS Genetics 4, e1000193

132 Gitcho, M.A. et al. (2008) TDP-43 A315T mutation in familial motor neuron disease. Annals of Neurology 63, 535-538

133 Van Deerlin, V.M. et al. (2008) TARDBP mutations in amyotrophic lateral sclerosis with TDP-43 neuropathology: a genetic and histopathological analysis. Lancet Neurology 7, 409-416

134 Kuhnlein, P. et al. (2008) Two German kindreds with familial amyotrophic lateral sclerosis due to TARDBP mutations. Archives of Neurology 65, 1185-1189

135 Corrado, L. et al. (2009) High frequency of TARDBP gene mutations in Italian patients with amyotrophic lateral sclerosis. Human Mutation 30, 688-394

136 Daoud, H. et al. (2009) Contribution of TARDBP mutations to sporadic amyotrophic lateral sclerosis. Journal of Medical Genetics 46, 112-114

137 Yokoseki, A. et al. (2008) TDP-43 mutation in familial amyotrophic lateral sclerosis. Annals of Neurology 63, 538-542 
138 Pamphlett, R. et al. (2008) TDP-43 neuropathology is similar in sporadic amyotrophic lateral sclerosis with or without TDP-43 mutations. Neuropathology and Applied Neurobiology 35, 222-225

139 Lemmens, R. et al. (2009) TDP-43 M311V mutation in familial amyotrophic lateral sclerosis. Journal of Neurology Neurosurgery and Psychiatry 80, 354-355

140 Del Bo, R. et al. (2009) TARDBP (TDP-43) sequence analysis in patients with familial and sporadic ALS: identification of two novel mutations. European Journal of Neurology 16, 727-732

141 Benajiba, L. et al. (2009) TARDBP mutations in motoneuron disease with frontotemporal lobar degeneration. Annals of Neurology 65, 470-473

142 Pikkarainen, M., Hartikainen, P. and Alafuzoff, I. (2008) Neuropathologic Features of Frontotemporal Lobar Degeneration With Ubiquitin-Positive Inclusions Visualized With Ubiquitin-Binding Protein p62 Immunohistochemistry. Journal of Neuropathology and Experimental Neurology 67, 280-298

143 Josephs, K.A. et al. (2008) Frontotemporal lobar degeneration with ubiquitin-positive, but TDP-43negative inclusions. Acta Neuropathologica 116, 159-167

144 Mackenzie, I.R. et al. (2008) Atypical frontotemporal lobar degeneration with ubiquitinpositive, TDP-43-negative neuronal inclusions. Brain 131, 1282-1293

145 Roeber, S. et al. (2008) TDP-43-negative FTLD-U is a significant new clinico-pathological subtype of FTLD. Acta Neuropathologica 116, 147-157

146 Holm, I.E. et al. (2007) A Reassessment of the Neuropathology of Frontotemporal Dementia Linked to Chromosome 3. Journal of Neuropathology and Experimental Neurology 66, 884-891

147 Uchikado, H. et al. (2005) Screening for neurofilament inclusion disease using alphainternexin immunohistochemistry. Neurology 64, 1658-1659

148 Cairns, N.J. et al. (2004) Clinical and neuropathologic variation in neuronal intermediate filament inclusion disease. Neurology 63, 1376-1384

149 Cairns, N.J. et al. (2004) alpha-internexin is present in the pathological inclusions of neuronal intermediate filament inclusion disease. American Journal of Pathology 164, 2153-2161

150 Yokota, O. et al. (2008) Basophilic inclusion body disease and neuronal intermediate filament inclusion disease: a comparative clinicopathological study. Acta Neuropathologica 115, 561-575

151 Roeber, S. et al. (2006) Neurodegeneration with features of NIFID and ALS-extended clinical and neuropathological spectrum. Brain Pathology 16, 228-234

152 Molina-Porcel, L. et al. (2008) Clinical and pathological heterogeneity of neuronal intermediate filament inclusion disease. Archives of Neurology 65, 272-275

153 Mackenzie, I.R. and Feldman, H. (2004) Neurofilament inclusion body disease with early onset frontotemporal dementia and primary lateral sclerosis. Clinical Neuropathology 23, 183-193

154 Josephs, K.A. et al. (2003) Neurofilament inclusion body disease: a new proteinopathy? Brain 126, 2291-2303

155 Josephs, K.A. et al. (2005) Extending the clinicopathological spectrum of neurofilament inclusion disease. Acta Neuropathologica 109, 427-432

156 Bigio, E.H. et al. (2003) Frontotemporal and motor neurone degeneration with neurofilament inclusion bodies: additional evidence for overlap between FTD and ALS. Neuropathology and Applied Neurobiology 29, 239-253

157 Cairns, N.J. et al. (2003) Patients with a novel neurofilamentopathy: dementia with neurofilament inclusions. Neuroscience Letters 341, 177-180

158 Mosaheb, S. et al. (2005) Neuronal intranuclear inclusions are ultrastructurally and immunologically distinct from cytoplasmic inclusions of neuronal intermediate filament inclusion disease. Acta Neuropathologica 110, 360-368

159 Uchikado, H. et al. (2006) Heterogeneous inclusions in neurofilament inclusion disease. Neuropathology 26, 417-421

160 Liu, Q. et al. (2004) Neurofilament proteins in neurodegenerative diseases. Cellular and Molecular Life Sciences 61, 3057-3075

161 Ching, G.Y. and Liem, R.K. (1991) Structure of the gene for the neuronal intermediate filament protein alpha-internexin and functional analysis of its promoter. Journal of Biological Chemistry 266, 19459-19468

162 Fliegner, K.H. et al. (1994) Expression of the gene for the neuronal intermediate filament protein alpha-internexin coincides with the onset of neuronal differentiation in the developing rat 
nervous system. Journal of Comparative Neurology 342, 161-173

163 Ching, G.Y. and Liem, R.K. (1998) Roles of head and tail domains in alpha-internexin's selfassembly and coassembly with the neurofilament triplet proteins. Journal of Cell Science 111, 321-333

164 Lariviere, R.C. and Julien, J.P. (2004) Functions of intermediate filaments in neuronal development and disease. Journal of Neurobiology 58, 131-148

165 Cairns, N.J. et al. (2004) alpha-Internexin aggregates are abundant in neuronal intermediate filament inclusion disease (NIFID) but rare in other neurodegenerative diseases. Acta Neuropathologica 108, 213-223

166 Momeni, P. et al. (2006) Mutation analysis of patients with neuronal intermediate filament inclusion disease (NIFID). Neurobiology of Aging 27, 778.e1-778.e6

167 Kusaka, H., Matsumoto, S. and Imai, T. (1993) Adult-onset motor neuron disease with basophilic intraneuronal inclusion bodies. Clinical Neuropathology 12, 215-218

168 Tsuchiya, K. et al. (2001) Familial amyotrophic lateral sclerosis with posterior column degeneration and basophilic inclusion bodies: a clinical, genetic and pathological study. Clinical Neuropathology 20, 53-59

169 Ishihara, K. et al. (2006) An autopsy case of frontotemporal dementia with severe dysarthria and motor neuron disease showing numerous basophilic inclusions. Neuropathology 26, 447-454

170 Hamada, K. et al. (1995) Dementia with ALS features and diffuse Pick body-like inclusions (atypical Pick's disease?). Clinical Neuropathology 14, 1-6

171 Munoz-Garcia, D. and Ludwin, S.K. (1984) Classic and generalized variants of Pick's disease: a clinicopathological, ultrastructural, and immunocytochemical comparative study. Annals of Neurology 16, 467-480

172 Van Gerpen, J.A. et al. (2008) Insights into the dynamics of hereditary diffuse leukoencephalopathy with axonal spheroids. Neurology 71, 925-929

173 Munoz-Garcia, D. and Ludwin, S.K. (1986) Adultonset neuronal intranuclear hyaline inclusion disease. Neurology 36, 785-790

174 Kertesz, A. et al. (2005) The evolution and pathology of frontotemporal dementia. Brain 128, 1996-2005
175 Forman, M.S. et al. (2006) Frontotemporal dementia: clinicopathological correlations. Annals of Neurology 59, 952-962

176 Mesulam, M. et al. (2008) Alzheimer and frontotemporal pathology in subsets of primary progressive aphasia. Annals of Neurology 63, 709-719

177 Munoz, D.G., Woulfe, J. and Kertesz, A. (2007) Argyrophilic thorny astrocyte clusters in association with Alzheimer's disease pathology in possible primary progressive aphasia. Acta Neuropathologica 114, 347-357

178 Uryu, K. et al. (2008) Concomitant TAR-DNAbinding protein 43 pathology is present in Alzheimer disease and corticobasal degeneration but not in other tauopathies. Journal of Neuropathology and Experimental Neurology 67, 555-564

179 Nakashima-Yasuda, H. et al. (2007) Co-morbidity of TDP-43 proteinopathy in Lewy body related diseases. Acta Neuropathologica 114, 221-229

180 Higashi, S. et al. (2007) Concurrence of TDP-43, tau and alpha-synuclein pathology in brains of Alzheimer's disease and dementia with Lewy bodies. Brain Research 1184, 284-294

181 Arai, T. et al. (2009) Phosphorylated TDP-43 in Alzheimer's disease and dementia with Lewy bodies. Acta Neuropathologica 117, 125-136

182 Amador-Ortiz, C. et al. (2007) TDP-43 immunoreactivity in hippocampal sclerosis and Alzheimer's disease. Annals of Neurology 61, 435-445

183 Ghidoni, R. et al. (2008) Low plasma progranulin levels predict progranulin mutations in frontotemporal lobar degeneration. Neurology 71, 1235-1239

184 Steinacker, P. et al. (2008) TDP-43 in cerebrospinal fluid of patients with frontotemporal lobar degeneration and amyotrophic lateral sclerosis. Archives of Neurology 65, 1481-1487

185 Foulds, P. et al. (2008) TDP-43 protein in plasma may index TDP-43 brain pathology in Alzheimer's disease and frontotemporal lobar degeneration. Acta Neuropathologica 116, 141-146

186 Tolnay, M. et al. (2000) A new case of frontotemporal dementia and parkinsonism resulting from an intron $10+3$-splice site mutation in the tau gene: clinical and pathological features. Neuropathology and Applied Neurobiology 26, 368-378 


\section{Further reading, resources and contacts}

\section{Publications}

Ballatore, C., Lee, V.M. and Trojanowski, J.Q. (2007) Tau-mediated neurodegeneration in Alzheimer's disease and related disorders. Nature Reviews Neuroscience 8, 663-672

A review of role of tau protein in neurodegeneration.

Buratti, E. and Baralle, F.E. (2008) Multiple roles of TDP-43 in gene expression, splicing regulation, and human disease. Frontiers in Bioscience 13, 867-878

This is an excellent review on the biology of TDP-43.

Ahmed, Z. et al. (2007) Progranulin in frontotemporal lobar degeneration and neuroinflammation. Journal of Neuroinflammation 4, 7

A review of progranulin function and role in FTD.

\section{Websites}

The website of the Association for Frontotemporal Dementias (AFTD), a US nationwide non-profit organisation whose mission is to promote and fund research into finding the cause and cure for FTD, provides information, education, and support to persons diagnosed with FTD and their families and caregivers; and educates physicians and allied health professionals about FTD:

http://www.ftd-picks.org/

The website from University of California, San Francisco provides information on clinical and research aspects of FTD and gives information on clinical trials:

http://memory.ucsf.edu/ftd/

The AD\&FTD Mutation database provides a current list of known FTLD gene mutations, including the number of affected families:

http://www.molgen.ua.ac.be/FTDmutations

\section{Features associated with this article}

\section{Figures}

Figure 1. Tau isoforms and tau gene mutations.

Figure 2. Pathological features in FTLD-tau.

Figure 3. TDP-43 and pathological spectrum of FTLD-TDP.

Figure 4. Pathological spectrum of FTLD-UPS.

Figure 5. Neuropathology of rare FTLD subtypes.

Table

Table 1. Recommended nomenclature for frontotemporal lobar degenerations.

\section{Citation details for this article}

Manuela Neumann, Markus Tolnay and lan R.A. Mackenzie (2009) The molecular basis of frontotemporal dementia. Expert Rev. Mol. Med. Vol. 11, e23, July 2009, doi:10.1017/S1462399409001136 